\title{
Extending the depth of field in a fixed focus lens using axial colour
}

Niamh Fitzgerald, Christopher Dainty, Alexander V. Goncharov

Niamh Fitzgerald, Christopher Dainty, Alexander V. Goncharov, "Extending the depth of field in a fixed focus lens using axial colour," Proc. SPIE 10590, International Optical Design Conference 2017, 105902A (27 November 2017); doi: $10.1117 / 12.2287600$

SPIE Event: International Optical Design Conference - IODC 2017, 2017, Denver, United States 


\title{
Extending the depth of field in a fixed focus lens using axial colour
}

\author{
Niamh Fitzgerald ${ }^{\mathrm{a}}$, Christopher Dainty ${ }^{\mathrm{b}}$, and Alexander V. Goncharov ${ }^{\mathrm{a}}$ \\ ${ }^{a}$ Applied Optics Group, School of Physics, National University of Ireland, Galway, Ireland \\ ${ }^{\mathrm{b}}$ FotoNation Ltd, Parkmore East Ind. Est, Ballybrit, Galway, Ireland
}

\begin{abstract}
We propose a method of extending the depth of field (EDOF) of conventional lenses for a low cost iris recognition front-facing smartphone camera. Longitudinal chromatic aberration (LCA) can be induced in the lens by means of dual wavelength illumination. The EDOF region is then constructed from the sum of the adjacent depths of field from each wavelength illumination. The lens parameters can be found analytically with paraxial raytracing. The extended depth of field is dependant on the glass chosen and position of the near object point.
\end{abstract}

Keywords: Chromatic aberration, Optical design, Iris imaging, Extended depth of field

\section{INTRODUCTION}

Smartphone camera lenses typically have low f-numbers for efficient light collection. Such f-numbers are associated with a small depth of focus $\Delta z$, where $\Delta z=2 \lambda(F \#)^{2}$. However, this is not a desirable property for fixed focus lenses as refocussing is not available.

The depth of field is a region in object space that produces a sharp image on a fixed position detector. For an object at infinity, the depth of field can be approximately calculated from a known depth of focus, $\Delta z$, and magnification:

$$
\Delta Z \approx \frac{\Delta z}{m^{2}}
$$

where $f$ is the focal length of the lens and $D$ is the diameter of the entrance pupil. For $f \ll l$ the longitudinal magnification of the lens can be approximated as the square of the transverse magnification. ${ }^{1}$ The magnification is expressed as $m=l^{\prime} / l \approx f / l$, then the approximate depth of field can be written as:

$$
\Delta Z=2 \lambda \frac{f^{2}}{D^{2}} \frac{l^{2}}{f^{2}}=2 \lambda \frac{l^{2}}{D^{2}}
$$

Unlike the symmetric depth of focus $\Delta z$, depth of field $\Delta Z$ is asymmetric. Equation 1 and 2 are approximate, as the general expressions for depth of focus and f-number are calculated for an object at infinity. For finite distances the relationships can be derived from paraxial raytracing.

Previous methods of extending the depth of field in imaging systems include computational algorithms such as coded apertures or introducing special optical elements to the system. The PSF and OTF of an optical system can be modified to be invariant of object position by employing phase masks for wavefront coding so that within the depth of field, these systems are diffraction limited ${ }^{2}{ }^{3}$ For an object that lies in a certain range of object distances, the optical system produces a known invariant PSF, which allows one to deconvolve the blurry image to restore its sharpness without need to estimate the object distance ${ }^{4}{ }^{5}$

Multiple Fresnel lenses can mimic the effect of a phase mask, where each Fresnel lens works in conjunction with the primary lens to produce a sharp image at discrete locations along the optical axis. ${ }^{6}$ This method can produce a sharper image with a larger depth of field when compared with a clear-aperture lens of the same

Further author information: E-mail: n.fitzgerald1@nuigalway.ie 
pupil size without the use of image post-processing. A combination of logarithmic aspheres together and postprocessing allows for a desired PSF to be generated. This PSF can be used to compute and fabricate an aspheric lens or a blurring phase filter. Either approach is then combined with a commercial lens. ${ }^{7}$ An electrically tuneable liquid lens has been shown to be capable of producing three different lens powers and increasing the spatial depth perception to twice that of a conventional endoscope without image post processing. ${ }^{8}$ An EDOF effect can be produced by introducing a variable focal objective. This approach requires restoration algorithms for deconvolving the captured image. ${ }^{9}$ A micro lens array can be positioned in front of the image plane that can transform a single lens into a multi-focus plenoptic camera yielding an extended depth of field. ${ }^{10}$ The plenoptic camera has a sub array of micro lenses divided into three types. Each type having different focal lengths which overlap and optimise the effective resolution in the extended depth of field region. Reconstruction algorithms are then used to retrieve the EDOF image.

Spherical aberration, introduced by spherical optics, can aid an increase in depth of field by inducing a rotationally symmetric blur which is invariant across the field. ${ }^{11}$ For an object that lies within the EDOF region, with the exact position unknown, the image can be deblurred by applying a mean monochromatic PSF. In this paper the lens is achromatic over the visible range however transverse chromatic aberration in the lens must be corrected for imaging in polychromatic light. Applications for extended depth of field using chromatic aberration has been implemented in confocal microscopy. ${ }^{12}$ This application takes advantage of the longitudinal chromatic aberration from stock aspheric lenses.

Induced axial colour and post-processing of the image with sharpness metrics across colour channels can allow for detecting various object distances. ${ }^{13}$ Each colour in the RGB channel corresponds to a particular object distance. A depth map can be generated such that each colour is reconstructed based on the filtering parameters assigned to the respective colour. In contrast to this approach, we introduce a method that also relies on intrinsic longitudinal chromatic aberration to extend the depth of field by means of a dual-wavelength illumination. In our approach a sharp image is obtained in real time without the use of computational intensive algorithms. The main goal is to design a low-cost lens in a front-facing camera in mobile phones for iris recognition. The cost-effective solution is obtained by avoiding refocussing systems with voice coils that can increase the cost of the camera module in a mobile phone by $10 \%$.

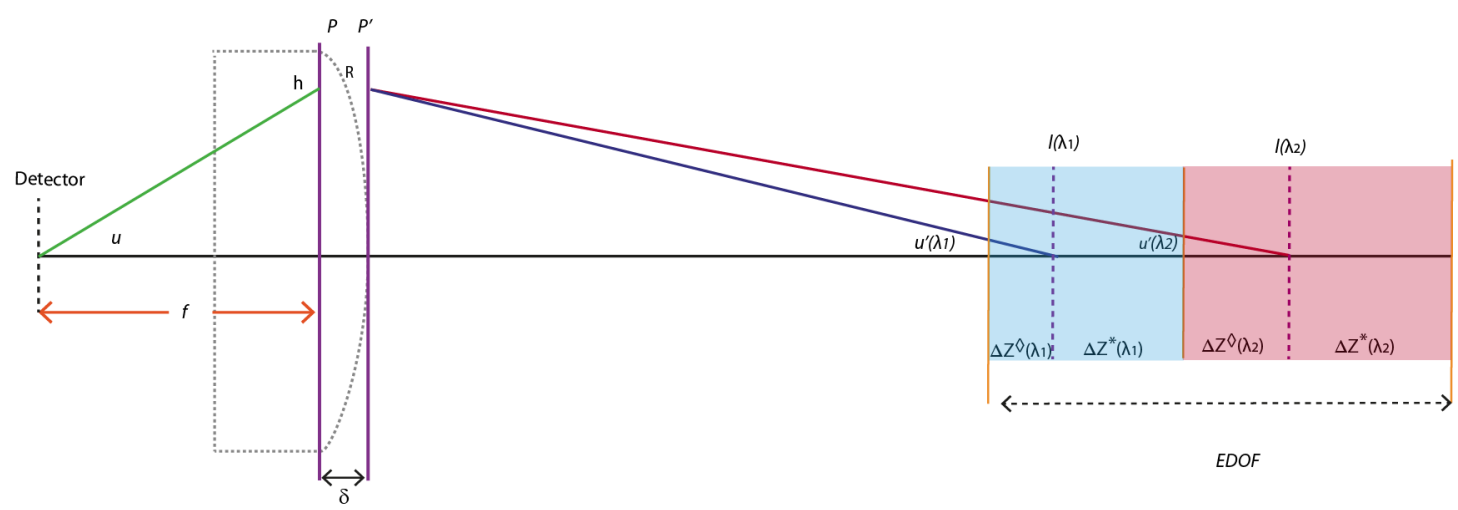

Figure 1. Diagram of reverse raytrace using the principle planes of a plano-convex singlet. The extended depth of field region is illustrated by the sum of the adjacet individual depths of field for $\lambda_{1}$ and $\lambda_{2}$ in blue and red respectively.

\section{METHODOLOGY}

Near infrared (NIR) illumination is chosen as the iris shows more detail in this region of the spectrum. ${ }^{14}$ In addition to the physical requirements of imaging the iris, there is a reduction in solar radiation in the NIR region. This helps to reduce the unwanted NIR background in daylight imaging.

Our methodology is demonstrated with a plano-convex singlet. This lens is selected as the location of the principle planes can be easily calculated and the mathematics is simplified. Figure 2 describes the lens design 
process. Dual wavelength illumination induces longitudinal chromatic aberration (LCA) in the lens. Using prior knowledge of the dispersive nature of the material used, one can reverse raytrace through the lens, that is trace from the detector into object space, and arrive at separate object points for each wavelength, $l\left(\lambda_{1}\right)$ and $l\left(\lambda_{2}\right)$, where for a refracting lens $l\left(\lambda_{1}\right)<l\left(\lambda_{2}\right)$, if $\lambda_{1}<\lambda_{2}$.

The initial design parameters are chosen as described in box 1 of Fig. 2: $\lambda_{1}, \lambda_{2}$, glass, BFD and $l\left(\lambda_{1}\right)$. The ray path for $\lambda_{1}$ and $\lambda_{2}$ can be traced from the detector through the singlet to form the wavelength dependant expression:

$$
u^{\prime}(\lambda)=1 / f(\lambda)+(n(\lambda)-1) / R=-1 / l(\lambda)
$$

Using equation 3, the radius of curvature $R$ can be calculated as a function of $\lambda_{1}$ and applied again to solve for the far object distance $\left.l_{(} \lambda_{2}\right)$ as a function of $\lambda_{2}$ as indicated by box 2 of Fig. 2. The separation of object points along the axis $\Delta l=l_{2}-l_{1}$ is dependant on the material and wavelengths chosen.

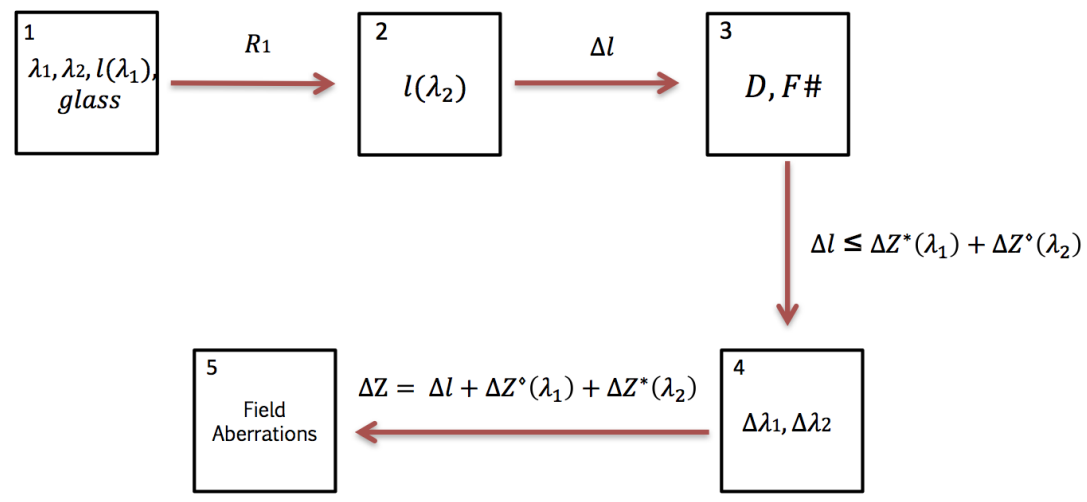

Figure 2. Flow chart describing the design process. The boxes are labelled $1-5$ following the design procedure.

Ensuring minimum defocus over the EDOF region, the depth of field for each source is required to adhere to a 'no-gap' condition, that is $\Delta Z^{*}\left(\lambda_{1}\right)+\Delta Z^{\diamond}\left(\lambda_{2}\right) \geq \Delta l$ as illustrated in Fig. 1. Selecting the correct f-number ensures the depth of field fulfil the condition, retaining a focused image over the EDOF region. The effect of bandwidth of the sources can be checked for intrinsic chromatic aberration such that it lies within the depth of field. Finally, aspheric surfaces are assigned to both surfaces of the lens to correct for field aberrations using exact raytracing.

\section{LENS EXAMPLES}

Standard N-SF5 glass is used to demonstrate our methodology. Two commercial NIR LEDs are chosen for iris illumination with wavelengths $\lambda_{1}=780 \pm 15 \mathrm{~nm}$ and $\lambda_{2}=870 \pm 20$. The near object position can be selected as a design parameter, in this application $l\left(\lambda_{1}\right)=300 \mathrm{~mm}$ being the distance between the iris and the vertex of the camera lens, which is a comfortable distance for a smartphone user. From Eq. 3 the radius is solved to be $R=-3.1821 \mathrm{~mm}$ and $l\left(\lambda_{2}\right)=432.36 \mathrm{~mm}$ yielding separation of object positions $\Delta l=132.36 \mathrm{~mm}$. Following the flow chart design process in Figure 2, the minimum f-number that fulfils the 'no gap' requirement is $F \# \geq 2.7$.

The maximum field angle achievable with diffraction limited performance is $6^{\circ}$ full field for a flat object surface as seen in Fig. 3. Spherical aberration and coma are corrected by the aspheric surfaces, however astigmatism is still present. Both surfaces of the lens are made aspheric and optimised. In order to increase the field of view of a singlet, we can assume that the object surface is a parabola and the radius of curvature for the object surface is optimised to minimise astigmatism over the field. The field of view is increased to $7.4^{\circ}$ full field with an object surface radius of $1.63 \mathrm{~mm}$. In addition to the extra field achieved using a parabolic object surface, the EDOF region is extended further by $60 \mathrm{~mm}$ towards the lens if the eye is decentered and follows the curvature of the radius for the object surface. 

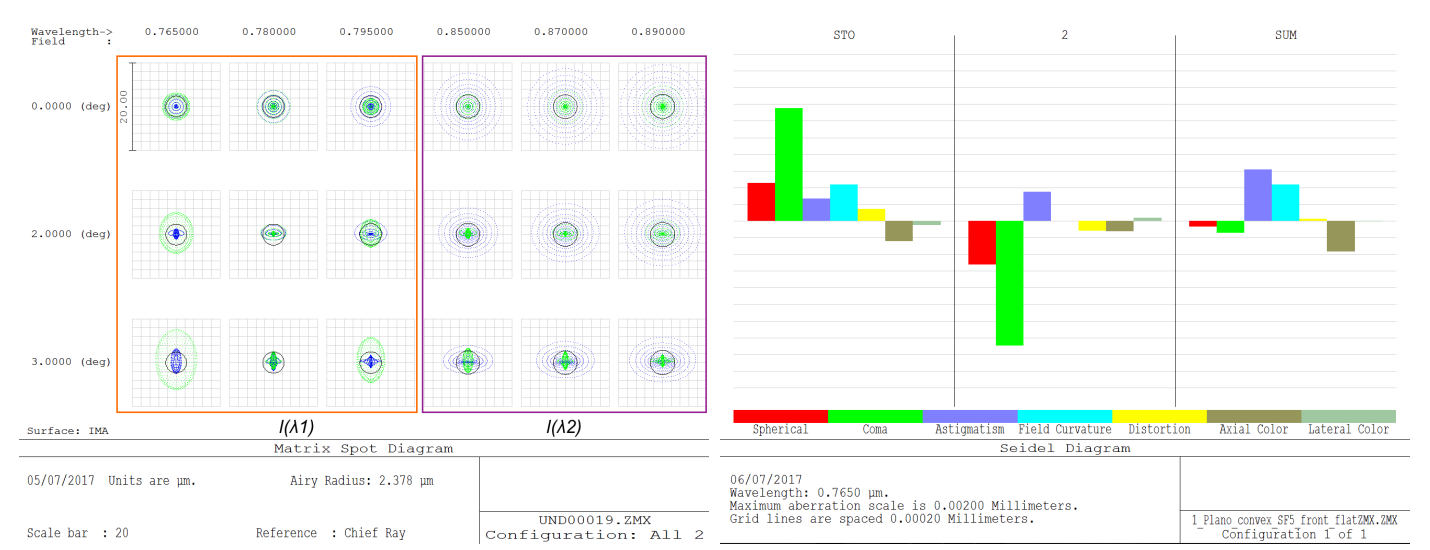

Figure 3. (a) Spot diagrams and (b) Seidel bar chart showing aberrations in image space for a high dispersing N-SF5 plano-convex singlet traced from a plane object surface. The spot diagrams enclosed in the orange box correspond to $\lambda_{1}$ being in focus at $l\left(\lambda_{1}\right)$ (blue spots in focus). The purple box shows the spot diagrams corresponding to $\lambda_{2}$ at $l\left(\lambda_{2}\right)$ (green spots in focus).

The additional depth of field attained by this decentering method is limited as the parabolic surface lies between the sagittal and tangential foci (when operating in reverse mode) and so the possibility of imaging the iris whole decreases with object height seen in Fig. 4. The iris (a) is positioned on axis at $l\left(\lambda_{1}\right)=300 \mathrm{~mm}$ with a full field of view of $7.4^{\circ}$. The iris at (b) is at $l\left(\lambda_{1}\right)=277 \mathrm{~mm}$ with object height of $10 \mathrm{~mm}$ and the iris at (c) is located at $l\left(\lambda_{1}\right)=240 \mathrm{~mm}$ at a height $15 \mathrm{~mm}$ from the optical axis. Figure 5 (a) shows the variation in $\Delta l$ changes with f-number for two plano-convex namely N-SF5 and an optical polymer E48R from the Zeon catalogue. The effect of near object position on the extended depth of field is shown in Fig. 5(b) for two plano-convex singlets made from N-SF5 glass and E48R plastic.

The effect of shape factor is investigated as a method of controlling $\Delta l$. Figure 6 shows four lens shape factors for N-SF5 singlets used in our study with accompanying spot diagrams and Seidel bar charts in each column. All singlets in this study have a parabolic object surface with optimised radius of curvature. For column (a) the back surface is concentric to the focal point and the radius of curvature of the front surface is calculated. The maximum field of view is $7.4^{\circ}$ with $\Delta l=126.1 \mathrm{~mm}$. Column (b) shows the plano-convex lens presented in the introductory example with an increased field of $7.4^{\circ}$. Column (c) is a reverse plano-convex lens which has an increased field of view of $12.8^{\circ}$ with $\Delta l=202.79 \mathrm{~mm}$. Finally (d) is optimised for two variable radii and converges to a meniscus lens with the largest field of view at $8^{\circ}$ and largest $\Delta l$ of $230 \mathrm{~mm}$.

(c)

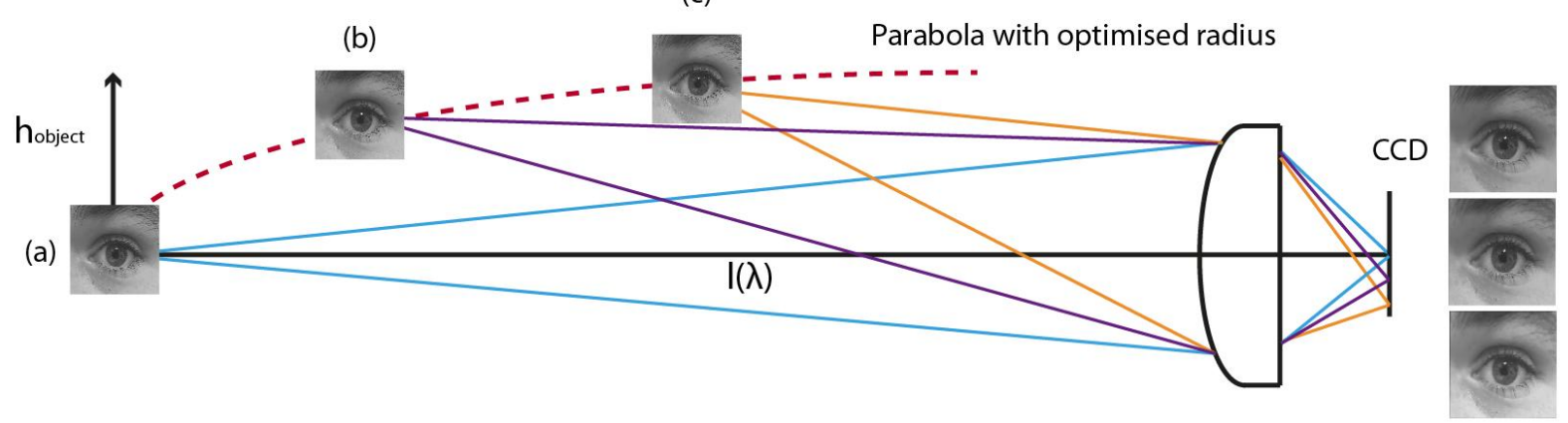

Figure 4. Decentering the iris along the parabolic surface in object space of a plano-convex singlet lens made of N-SF5 glass. The possibility of imaging the iris in whole becomes more challenging with increasing object height, $h_{\text {object }}$. Each iris in object space has a corresponding image simulated with Zemax. 

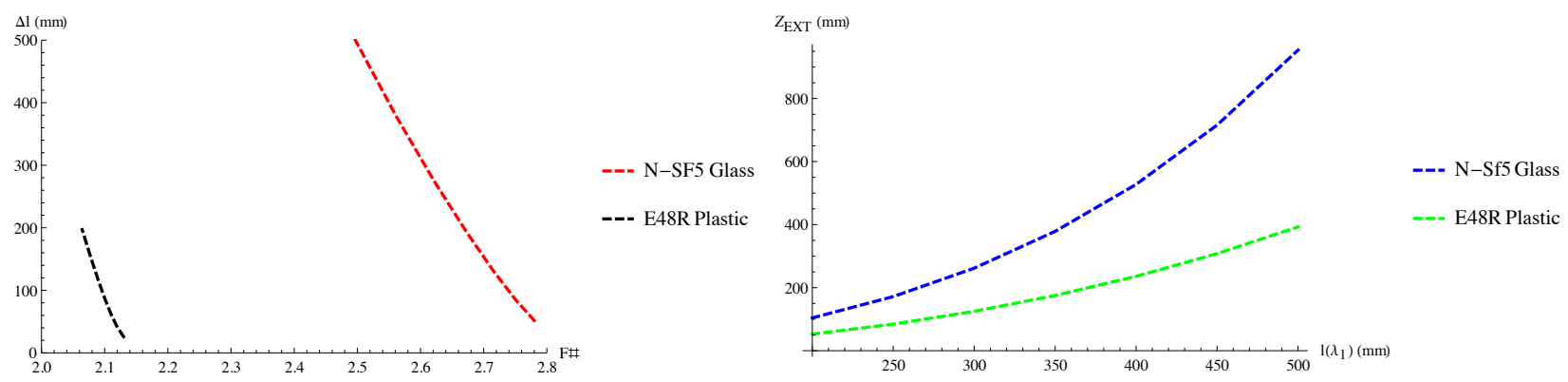

Figure 5. The variation of (a) $\Delta l$ with $F \#$ and (b) the change of extended depth of field (EDOF) with near object position $l\left(\lambda_{1}\right)$ for N-SF5 glass and E48R optical plastic.

As spherical aberration and coma are corrected by aspheric surfaces, astigmatism is minimised by a parabolic object surface, we can introduce shape factor as an approach for adjusting $\Delta l$. The concentric singlet can offer a modest decrease of $\Delta l$ having a shape factor of 3.35 with one surface contributing to refraction while the meniscus lens, optimised for minimum rms wavefront, induced approximately twice the amount of $\Delta l$ than the plano-convex lens with shape factor of -1.30 . It can be noted that an increase of $\Delta l$ results in an increase in the lens f-number.

For the object surface is modelled as a parabola, each singlet has an optimum radius of curvature corre-

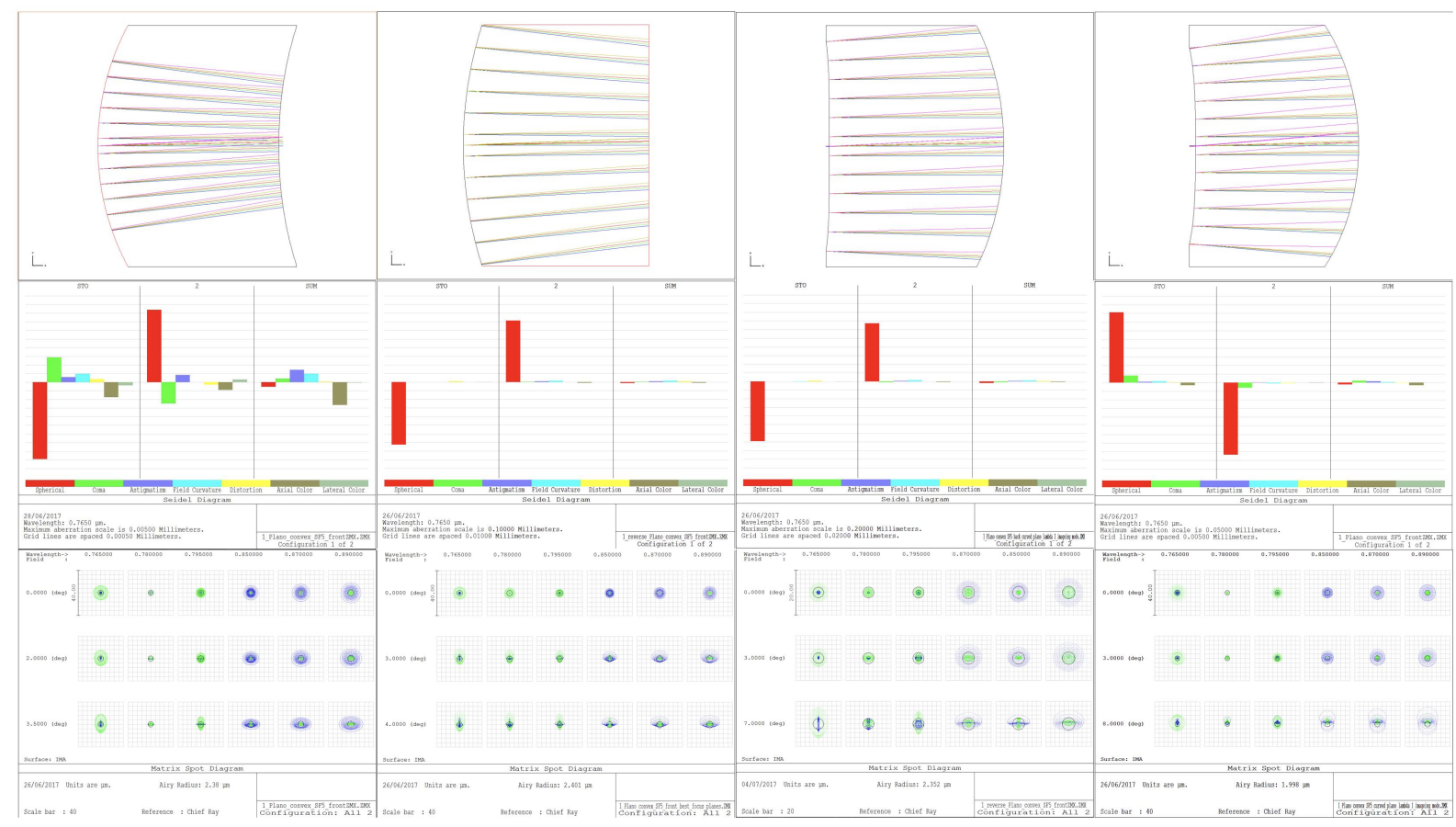

(a)

(b)

(c)

(d)

Figure 6. Singlet lenses made from N-SF5 glass with varying shape factor: (a) concentric surface to the focal point, (b) plano-convex, (c) reverse plano-convex and (d) meniscus singlets. Accompanying Seidel aberrations and spot diagrams follow vertically. 
sponding to the field curvature in the lens. The optimum radius for the object surface for each singlet lens is shown Fig. 7 indicating that the plano-convex lens has the capability to extend the EDOF region further into the region close to the lens which proves is the most difficult region to reach.

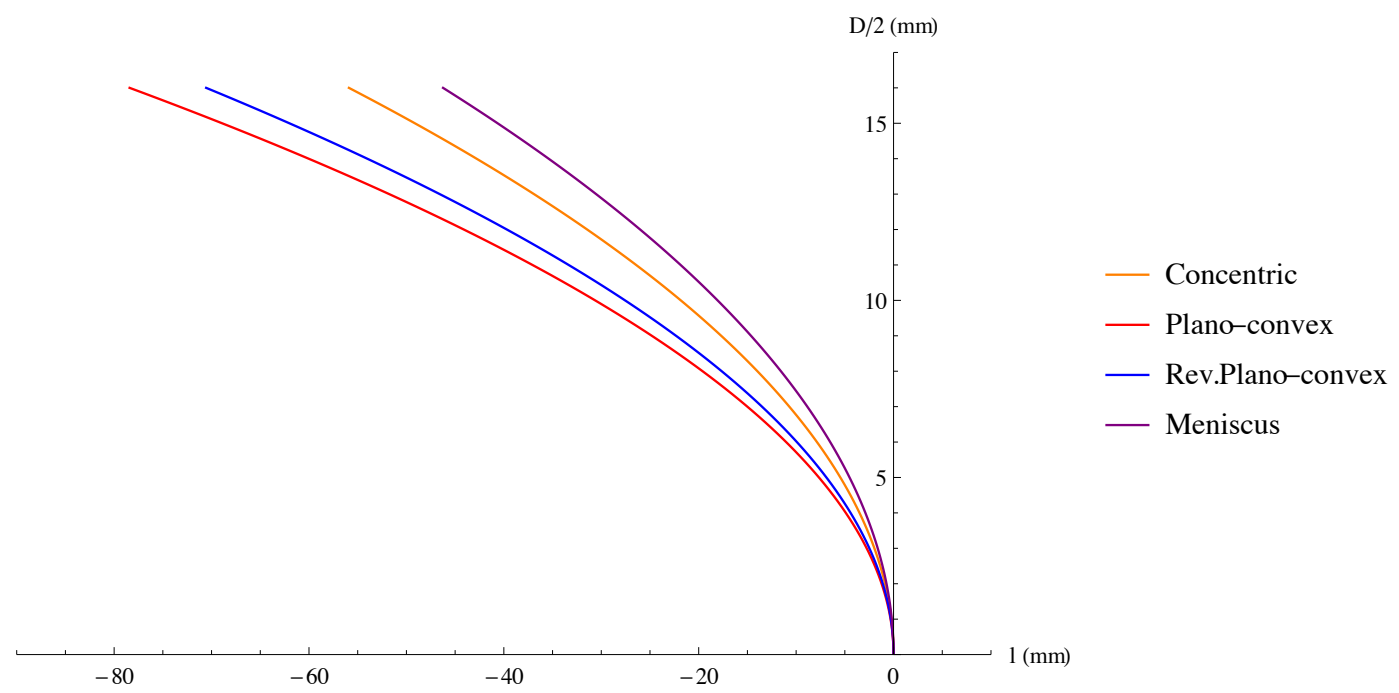

Figure 7. Optimised radius for Parabolic object surface for N-SF5 singlet lenses with different shape factor.

\begin{tabular}{|r|r|r|r|r|}
\hline Singlet & Concentric & Plano-Convex & Rev Plano-Convex & Meniscus \\
\hline Radius (mm) & 2.29 & 1.63 & 1.81 & 2.77 \\
\hline
\end{tabular}

Table 1. Optimal radius of curvature for each singlet lens with a parabolic object surface

\section{CONCLUSION}

We have presented a novel method of increasing the depth of field in smartphone cameras using dual wavelength illumination. Shape factor can introduce a small variation in $\Delta l$. However, for a large reduction in $\Delta l$, plastic lenses should be chosen as we have shown that a competitive f-number can be achieved at the expense of a reduction in EDOF.

\section{ACKNOWLEDGMENTS}

This research is funded under the SFI Strategic Partnership Program by Science Foundation Ireland (SFI) and FotoNation Ltd. Project ID: 13/SPP/I2868 on Next Generation Imaging for Smartphone and Embedded Platforms.

\section{REFERENCES}

[1] Gross, H., Dörband, B., and Müller, H., [Handbook of optical systems], vol. 1, Wiley Online Library (2005).

[2] Dowski, E. R. and Cathey, W. T., "Extended depth of field through wave-front coding," Applied Optics 34(11), 1859-1866 (1995).

[3] Chi, W., Chu, K., and George, N., "Polarization coded aperture," Optics express 14(15), 6634-6642 (2006).

[4] Nagahara, H., Kuthirummal, S., Zhou, C., and Nayar, S. K., "Flexible depth of field photography," in [European Conference on Computer Vision], 60-73, Springer (2008).

[5] Yokoya, R. and Nayar, S. K., "Extended depth of field catadioptric imaging using focal sweep," in [Proceedings of the IEEE International Conference on Computer Vision], 3505-3513 (2015). 
[6] Ben-Eliezer, E., Marom, E., Konforti, N., and Zalevsky, Z., "Experimental realization of an imaging system with an extended depth of field," Appl. Opt. 44, 2792-2798 (May 2005).

[7] George, N. and Chi, W., "Extended depth of field using a logarithmic asphere," Journal of Optics A: Pure and Applied Optics 5(5), S157 (2003).

[8] Chen, H.-S. and Lin, Y.-H., "An endoscopic system adopting a liquid crystal lens with an electrically tunable depth-of-field," Optics express 21(15), 18079-18088 (2013).

[9] Liu, S. and Hua, H., "Extended depth-of-field microscopic imaging with a variable focus microscope objective," Opt. Express 19, 353-362 (Jan 2011).

[10] Perwass, C. and Wietzke, L., "Single lens 3d-camera with extended depth-of-field.," in [Human Vision and Electronic Imaging], 17 (2012).

[11] Mouroulis, P., "Depth of field extension with spherical optics," Opt. Express 16, 12995-13004 (Aug 2008).

[12] Olsovsky, C., Shelton, R., Carrasco-Zevallos, O., Applegate, B. E., and Maitland, K. C., "Chromatic confocal microscopy for multi-depth imaging of epithelial tissue," Biomedical optics express 4(5), 732-740 (2013).

[13] Tisse, C.-L., Nguyen, H. P., Tessières, R., Pyanet, M., and Guichard, F., "Extended depth-of-field (edof) using sharpness transport across colour channels," Novel Optical Systems Design and Optimization XI, San Diego, CA, USA 7061, 706105-1 (2008).

[14] Atchison, D. A. and Smith, G., [Optics of the human eye], Butterworth-Heinemann Oxford (2000). 